\title{
Bilateral primary osteoarthritis of elbow: A case report with review of literature.
}

\author{
Sandesh S. Thete, Gajanan K. Deshmukh, Dwarkadas G. Tapadiya
}

\begin{abstract}
:
Introduction: Primary osteoarthritis $(O A)$ of the elbow is a rare condition, characterized by painful stiffness, mechanical symptoms, and the presence of hypertrophic osteophytes. Elbow osteoarthritis typically affects middle-aged men who engage in strenuous manual activity. We hereby present a case of bilateral primary elbow osteoarthritis and discuss its various treatment options.

Case report: A 45 years old male patient presented to outpatient department with complaints of pain in both elbows since one year. There was no history of previous trauma or other complaints pertaining to elbow. He was a manual laborer working in farms. He had tenderness on deep palpation of elbow. All movements at elbow (extension, flexion, supination and pronation) were terminally restricted. X-rays of both elbows revealed osteoarthritis of elbow. After thorough discussion of treatment options with patient, he opted for non-operative management.
\end{abstract}

Key massage: It requires a high index of suspicion to diagnose primary OA of elbow. Best treatment option involves capsular release and removal of impinging osteophytes but non-operative treatment remains the first step in the early management of elbow osteoarthritis.

Key words: primary elbow osteoarthritis, bilateral, management Case Report

\section{Introduction}

Elbow osteoarthritis, although rare, is a disabling condition because of pain and loss of motion. Osteoarthritis has been traditionally taught to be primary or secondary. Osteoarthritis is said to be primary when no anatomical or pathological etiology can be defined as a cause for it. Primary OA mainly affects weight bearing joints of the lower extremity. The common assertion that the elbow is not a weight-bearing joint should not suggest that the elbow does not bear load. We hereby present a case of bilateral primary elbow osteoarthritis and discuss its clinical and radiological features and various treatment options.

\section{Case Report-}

A 45 years male patient presented to outpatient department with pain in both elbows since one year. He was a manual laborer working in farms since 20 years. The pain was gradual in onset, progressive, diffuse, dull aching, aggravated by activity and relieved by rest without any diurnal variations. The pain was associated with stiffness of both elbows. There was no history of previous trauma to elbow. There was no significant past medical or surgical history. He was non diabetic. On local examination there was tenderness on deep palpation. Crepitus was felt with movements of elbows. There was restriction of both active and passive movements of both elbows with bony block at the end of movement. The arc of movements was $120^{\circ}$ with flexion $140^{\circ}$ and extension $20^{\circ}$ on both sides.

On taking Standard antero-posterior and lateral radiographs of the elbow, it showed evidence of osteoarthritis of both elbows. The radiographs show osteophytes but well-preserved ulnohumeral and radiocapitellar joint spaces, which are characteristic of primary osteoarthritis. It also revealed anterior and medial osteophyte involving the coronoid process and a posteromedial osteophyte on the olecranon process. Corresponding osteophytes on the humeral side were found in the coronoid and olecranon fossae. Considering the clinical and radiological findings a diagnosis of bilateral primary osteoarthritis was made. After discussing the treatment options with patient he opted for non operative management.

\section{Discussion-}

The elbow is not a weight-bearing joint, and the arthritis is frequently asymptomatic. However the common assertion that the elbow is not a weight-bearing joint should not suggest that the elbow does not bear load. Chadwick and Nicol reported that resultant forces of up to two times body weight could occur across the ulnohumeral joint during motions commonly seen in occupational duties, such as lifting, moving, and placing 2$\mathrm{kg}$ weights ${ }^{1,2}$. Dynamic loading, as seen during throwing or heavy pounding, produces forces of more than six times the body weight ${ }^{3}$. 
Symptomatic elbow osteoarthritis is characterized by pain and loss of motion. The etiology of osteoarthritis of the elbow has been a subject of great debate. Most early reports described an association between strenuous manual labor (specifically the use of pneumatic tools) and the development of elbow osteoarthritis. Trauma, osteochondritis dissecans, synovial chondromatosis ${ }^{4}$, and valgus extension overload ${ }^{3}$ coronoid fracture are few of the causes of secondary osteoarthritis. The valgus extension overload syndrome is a well-known cause of osteoarthritis in overhead throwers ${ }^{3}$.

Vocation is important, as patients with primary osteoarthritis are frequently employed in a job that requires strenuous manual labor. The degree of pain and disability varies among patients and is affected by handedness and vocational and recreational demands. The duration of the symptoms, location of the pain, mechanical symptoms, presence of pain at rest or at night, and character and quality of the pain are important aspects of the history. Many patients with osteoarthritis report pain at the end ranges of motion rather than at the mid-range because of osteophyte impingement ${ }^{5}$.

Visual inspection shows no deformity with terminal restriction of movements. Palpation in the lateral soft spot, which is located in the center of a triangle on the lateral aspect of the elbow and is bordered by the tip of the olecranon, the lateral epicondyle, and the radial head, may demonstrate tenderness and effusion. Motion is examined in flexion-extension and pronation-supination. Crepitus is often present during motion of arthritic elbows. It is important to note whether pain is present only at the end points of motion or throughout the arc of motion. Osteophyte impingement causes pain at the limits of forced extension or flexion, but large osteochondral lesions cause pain in the mid-range of motion. Loss of motion in all planes is common. A thorough neurovascular examination should be performed during the initial evaluation. Examination of the ulnar nerve is particularly important. Ulnar neuropathy may be present, but, even more importantly, any history or evidence of previous surgical transposition of the nerve influences preoperative surgical planning and the surgical approach ${ }^{5}$.

Standard anteroposterior and lateral radiographs of the elbow are usually sufficient for the initial evaluation. Osteoarthritis of the elbow differs from that of other joints in that it is characterized not necessarily by loss of joint space but rather by osteophyte formation and capsular contracture, with or without the presence of loose bodies. Up to $30 \%$ of loose bodies are not detected on plain radiographs. Additional imaging studies are not routinely necessary for preoperative planning ${ }^{6}$.

Treatment can be either conservative or operative; however conservative treatment is the first line of management. Rest, anti-inflammatory medication, and long-term activity modification are the essential components of the conservative treatment plan. It is important to modify activity, which is thought to be associated with the disease etiology, but this is difficult for most patients with strenuous work demands. The judicious administration of intra-articular steroids and anesthetics can relieve pain and may improve the ability to perform range-of-motion exercises. However, injections are unlikely to provide long-term relief for patients with advanced disease and should be restricted in younger patients with a preserved joint space. A formal supervised program of physical therapy is not routinely required for the non-operative treatment of elbow osteoarthritis?.

Osteoarthritis of elbow is itself an indication for surgery, especially when conservative management is exhausted. The surgical management can divided into non-prosthetic and prosthetic options.

\section{Nonprosthetic Management}

Joint débridement, capsular release, and removal of osteophytes - a procedure termed ulnohumeral arthroplasty is indicated when loss of motion is the predominant clinical finding. Ideal candidates for this procedure are young and active and include those who have mechanical symptoms, pain at the end range of motion, and/or moderate stiffness and who have exhausted nonoperative treatment options ${ }^{8,10}$. Postoperative ulnar neuropathy can complicate ulnohumeral arthroplasty ${ }^{10}$.

The Outerbridge procedure- The Outerbridge procedure, as popularized by Kashiwagi ${ }^{9}$, was designed to remove loose bodies and hypertrophic osteophytes through a posterior approach and a fenestration of the olecranon fossa.

Arthroscopic Osteocapsular Arthroplasty- Arthroscopic ulnohumeral (or osteocapsular) arthroplasty has been introduced as a method to address the various pathologic features in an arthritic elbow while reducing the postoperative morbidity associated with a large incision and exposure of the joint ${ }^{11}$. The arthroscopic procedure similarly involves capsular release, removal of marginal osteophytes, and joint débridement with removal of loose bodies. Arthroscopic osteocapsulararthroplasty has several potential advantages over an open procedure.

\section{Prosthetic management-}

Total Elbow Arthroplasty- Total elbow arthroplasty is rarely indicated for the treatment of primary elbow osteoarthritis. Elbow osteoarthritis typically affects younger or middle-aged, active men employed in 
high-demand jobs. These patients are not candidates for total elbow arthroplasty because of concerns about prosthetic longevity ${ }^{12}$. Currently, total elbow arthroplasty is indicated for patients who are older than sixty-five years of age and have low activity levels and pain throughout the range of motion or substantial deficits of motion and for whom the previously discussed interventions have failed ${ }^{13}$.

\section{References}

[1]. Chadwick EK, Nicol AC. Elbow and wrist joint contact forces during occupational pick and place activities. J Biomech. 2000;33: 591-600.

[2]. An KN, Morrey BF. Biomechanics of the elbow. In: Morrey BF, editor. The elbow and its disorders. 3rd ed. Philadelphia: WB Saunders; 2000. p 43-60.

[3]. Schmitt H, Hansmann HJ, Brocai DR, Loew M. Long term changes of the throwing arm of former elite javelin throwers. Int J Sports Med. 2001;22: 275-279.

[4]. Bauer M, Jonsson K, Josefsson PO, Linden B. Osteochondritis dissecans of the elbow. A long-term follow-up study.ClinOrthopRelat Res. 1992;284: 156-60.

[5]. Stanley D. Prevalence and etiology of symptomatic elbow osteoarthritis. J Shoulder Elbow Surg. 1994;3: 386-9.

[6]. Minami M. Roentgenological studies of osteoarthritis of the elbow joint. Nippon SeikeigekaGakkaiZasshi. 1977;51: 1223-36. Japanese.

[7]. Gregory D. Gramstad ; Leesa M. Galatz Management of Elbow Osteoarthritis J Bone Joint Surg Am, 2006 Feb 01;88(2):421-430

[8]. Wada T, Isogai S, Ishii S, Yamashita T. Debridement arthroplasty for primary osteoarthritis of the elbow. J Bone Joint Surg Am. 2004;86: 233-41.

[9]. Kashiwagi D. Intra-articular changes of the osteoarthritic elbow, especially about the fossa olecranon. JpnOrthop Assn. 1978;52: 1367-82.

[10]. Oka Y, Ohta K, Saitoh I. Debridement arthroplasty for osteoarthritis of the elbow. ClinOrthopRelat Res. 1998;351: 127-34.

[11]. O'Driscoll SW. Arthroscopic treatment for osteoarthritis of the elbow.OrthopClin North Am. 1995;26: 691-706.

[12]. Tsuge K, Mizuseki T. Debridement arthroplasty for advanced primary osteoarthritis of the elbow. Results of a new technique used for 29 elbows. J Bone Joint Surg Br. 1994;76: 641-6.

[13]. Goldberg VM, Figgie HE 3rd, Inglis AE, Figgie MP. Total elbow arthroplasty. J Bone Joint Surg Am. 1988;70: 778-83.
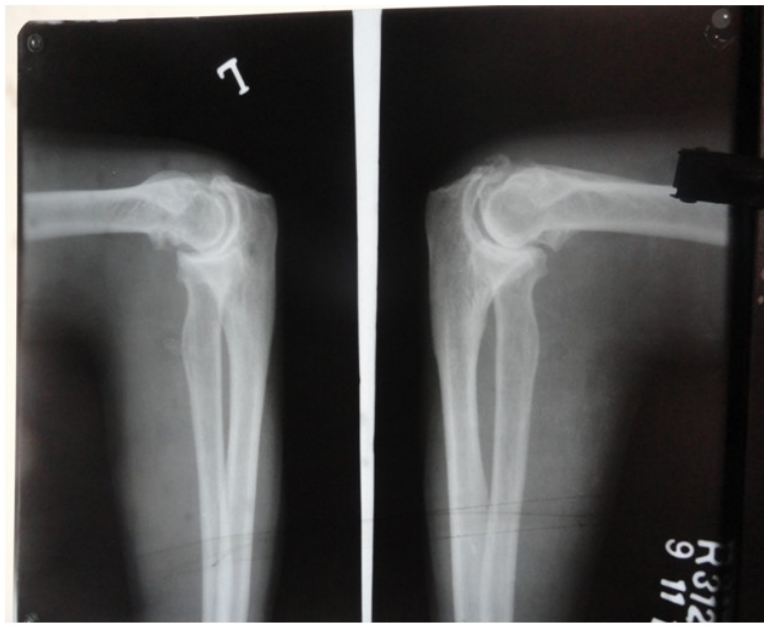

Figure 1-: Lateral radiograph of both elbows showing osteophytes in coronoid fossa and olecrenon process with well maintained joint space.

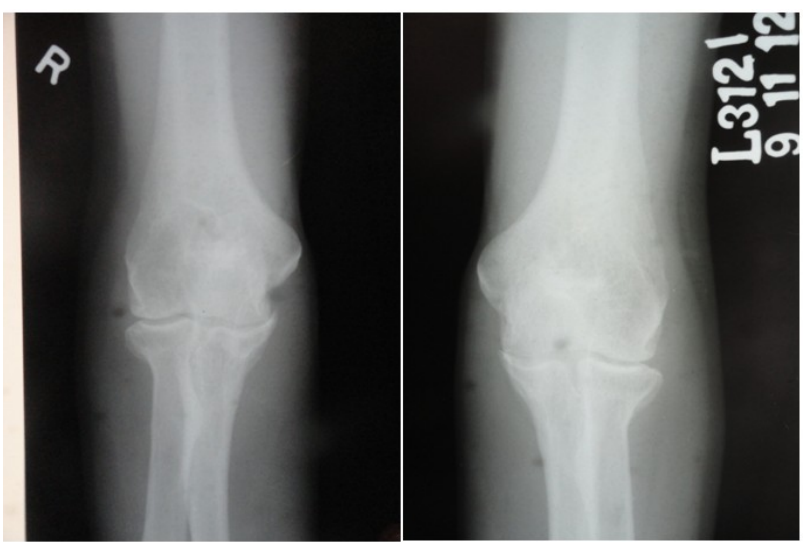

Figure 2-: AP Xray of both elbow showing well preserved symmetrical joint space. Note the obscured olecrenon fossa due to presence of osteophytes. 